\title{
Cultural ecosystem services in the UK: lessons on designing indicators to inform management and policy
}

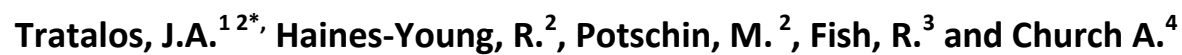 \\ ${ }^{1}$ CVERA, School of Veterinary Medicine, University College, Dublin, Belfield, Dublin 4, Ireland \\ * Corresponding author: Jamie.Tratalos@ucd.ie \\ ${ }^{2}$ CEM, School of Geography, University of Nottingham, Nottingham, NG7 2RD \\ ${ }^{3}$ Department of Politics, University of Exeter, Exeter, EX4 4RJ \\ ${ }^{4}$ Environment and Technology, University of Brighton, Brighton, BN2 4GJ
}

\begin{abstract}
We explore the degree to which a useful and cost-effective set of cultural ecosystem services (CES) indicators can be produced at different spatial scales in the UK, using readily available data. We place this within the conceptual framework developed for the understanding of CES produced by the recent UK National Ecosystem Assessment (UK NEA) and its Follow-on.

We examine a set of 'supply-side' stock indicators, involving the calculation of the percentage cover of environmental places such as woodlands, country parks and private gardens. Although stock indicators can be used to measure the potential of different localities to deliver CES, the accessibility of these environmental places to local populations also needs to be understood. We illustrate this with indicators based on access to four types of environmental space: ancient woodland, country parks, nature reserves and areas with natural cover. We illustrate how both the stock- and access-based indicators could be used to benchmark Local Authority Districts (LADs) across the whole of the UK.

We explore how a range of indicators can be developed from readily available information to compare the quality of environmental places at a more local level, using The City of Nottingham LAD as an example. We also examine the potential to use a household survey, the UK Monitor of Engagement with the Natural Environment (MENE), to estimate demand for certain types of cultural practice and environmental place, and to use relationships revealed in this information to estimate the degree to which supply and demand for these practices and places match one another.
\end{abstract}

Keywords: Cultural Ecosystem Services, indicators, benchmarking, National Ecosystem Assessment. 


\section{Introduction}

Within the spectrum of Ecosystem Services, it has proved particularly difficult to create practical, cost effective indicators for Cultural Ecosystem Services (CES) (Hernández-Morcillo et al., 2013). This partly reflects the characterisation of CES as an intangible and interpretative realm of ecosystem assessment and raises significant challenges for a field where the quantitative measurement of ecosystem services is considered central to their visibility within decision making. In this study we take a fresh look at the problem of creating indicators for CES, arguing that simple measures can be created from publicly available data that can help managers and decision makers to take CES into account.

\subsection{Cultural ecosystem indicators: the need to measure services, not benefits}

CES were described by the Millennium Assessment as 'the nonmaterial benefits people obtain from ecosystems through spiritual enrichment, cognitive development, reflection, recreation, and aesthetic experience" (MA, 2005). This definition has been criticised as it conflates services with the benefits they provide (Chan et al., (2011, 2012). One consequence of this is that cultural ecosystem services tend to be viewed as an intangible realm where applications of quantitative methods appear inappropriate or highly contextspecific. In the UK National Ecosystem Assessment (NEA) and its Follow-on (NEAFO) a distinction is made between the benefits provided by CES and the Environmental Spaces and the Cultural Practices undertaken within them which enable their delivery (Church et al., 2011, 2014). Although 'recreation', for example, had often been identified as a 'cultural ecosystem service' in studies that followed the MA, it was argued that it, together with spiritual, aesthetic and educational outcomes, is best regarded as a benefit or 'cultural good'. It was proposed that we might more usefully think of the bio-physical spaces in which these cultural benefits were obtained as representing 'final' cultural ecosystem services. A similar argument has been made in the development of the Common International Classification of Ecosystem Services (CICES) (Haines-Young and Potschin, 2013). The NEA conceptual framework thus envisages that cultural ecosystem services are delivered through 'environmental settings' or 'environmental spaces', that can be recognised by the range of cultural practices that take place within them. No strict typology has been derived for environmental spaces, but they include areas distinguished by natural or semi-natural vegetation, such as woods or moorland, as well as areas specifically designed for outdoor recreation, such as city and country parks, and recreation grounds, and areas designed for other purposes but which may also deliver CES, such as fields, canal tow paths, allotments and cemeteries. Cultural practices are the activities in which people engage in environmental spaces. These practices range from such things as going for a walk, meeting friends, watching wildlife and tending an allotment, and a variety of different cultural practices that may participated in by visitors within the same environmental place.

During the UK NEA Follow-on, this conceptual framework has been further refined by considering in detail the role of cultural values and the specific benefits provided when environmental spaces, cultural practices and cultural values come together. Environmental spaces are essentially distinct geographical entities, referred to as settings in the earlier NEA, that are distinguished by their potential to deliver CES. They both enable, and are in turn shaped by, cultural practices, which may be physical (embodied), textual (mediated) 
and/or linguistic (discursive) in form. Again, cultural practices reflect and constitute cultural values and are a discernible way that culture can be said to manifest itself, both at particular moments in time (e.g. through recreational activity) and as part of a broad cultural realm of lived experience (e.g. through expression of a whole 'way of life') (Williams, 1983).

The distinction between services and benefits is helpful for the development of CES indicators, as it suggests that measurement of the availability and quality of the natural environment, and the measurement of what is undertaken in it, can form the basis for measuring CES. This is arguably easier to achieve than directly measuring benefits, which are often intangible and hard to quantify.

\subsection{Review of existing indicator research}

The nature of the problem is illustrated by the fact that relatively little has been done to develop robust indicators for CES. In a review, Hernández-Morcillo et al. (2013) found only 38 of 344 indicators in the MA corresponded to cultural services, and noted that Rey Benayas et al. (2009) did not find a single study which measured Cultural Ecosystem Services explicitly, within a meta-analysis of 524 indicators of biodiversity and ecosystem services from 89 restoration assessments. In total Hernández-Morcillo et al. (2013) were able to examine 70 CES indicators, of which $54 \%$ were for recreation and ecotourism. Only $23 \%$ of the studies used spatially explicit information. CES indicators have most often been restricted to capturing visitor rates at specific sites as measures of demand (Plieninger et al., 2013) and do not, therefore, provide a basis for comparisons or benchmarking between locations. Studies undertaken for the Secretariat of the Convention on Biological Diversity noted that indicators of ecosystem services often focussed on purely provisioning services and often studies only used recreation and tourism as a measure of CES (UNEP-WCMC 2011).

Where spatial quantitative assessments have been made, these have mainly been in the form of in-depth consultative studies with local communities (see, for example, Balram et al. (2005), Brown \& Raymond (2007), Bieling \& Plieninger (2013), Plieninger et al. (2013)). Such localised studies are based on an understanding that CES are only intelligible in terms of an interaction between the natural environment and the cultural history and individual beliefs, preferences and values of a local population, and that there is a lack of knowledge of the way in which environmental spaces and the cultural practices which can be conducted in them affect benefit in a local context, and by what criteria the quality of different locations should be judged locally. For example, Jorgensen et al. (2007) found that for elderly age groups the degree to which they felt safe, as well as appreciation of links with the past and immersion in nature, may be relatively more important determinants of whether these groups enjoyed visiting urban woodlands, when compared with younger age groups. The same agricultural landscape may mean different things to those who have farmed it for generations compared to those who are enjoying it for it associations with a famous author.

Measuring CES is therefore not as straightforward as it often is for other ecosystem services, where there is often a clearer relationship between the quality of services provided and a measurable entity, such as amount of carbon sequestered by the trees in forest, or the number of species the same forest supports. The CES offered by a forest depend not only on the physical characteristics of the forest, but also on the importance it has for the local 
human population, how many people visit it, what they do there and the values they bring to the experience. Furthermore, because people enjoying CES often do so as part of a journey, it is difficult to ascribe specific benefits to specific locations or indeed to define these locations - a typical rural walk, for example, may take in a variety of habitats and environmental features, some of which are walked over or within, and others which are observed from afar.

In addition to the difficulty of adequately measuring such complex relationships in the local context, the benefits deriving from CES themselves have been divided into a number of different elements by the MA, which have been broadly accepted elsewhere: Spiritual and Religious, Recreation and Tourism, Aesthetic, Inspirational, Sense of Place, Cultural Heritage, Education (MA., 2005, Hernandez-Morcillo et al., 2013, Bieling \& Plieninger, 2013, Chan et al., 2011)). In practice, it is extremely difficult to disentangle these strands from one another as a single interaction with nature might involve aspects of all of them (Church et.al 2011).

In the face of such challenges, one approach might be to restrict attempts to measure CES to to fine-scale localised studies where research resources were available to conduct indepth investigations. Different policy and management initiatives are likely to wish to create their own indicators to answer the specific questions that face them, and it may therefore be better to restrict the creation of indicators for CES to ad hoc cases where the resources are available to derive a set of indicators to address the information needs of a specific programme or local initiative. However, there is a risk that complex localised measures of CES produced in this manner may fail to match criteria identified as important for an environmental indicator, such as the promotion of simplification and quantification, the need to be readily understandable, to use information that is readily available, to be costeffective, be quick to produce, and able to facilitate comparisons between different geographical areas (Environmental Challenge Group, (1995), Anderson, (1991), CSIRO (1998), Audit Commission (2005).

Another approach would be to acknowledge that, given the current state of our knowledge and the resources available for research, the measurement of CES would inevitably be imprecise, but that we should investigate whether cost effective indicators, using currently available data, could be produced which may at least partly address the information needs of those concerned with making policy or management decisions connected with CES. Although the resulting set of indicators may not have the depth of those produced for smaller scale studies, the use of data available at a country-wide level would mean that they would make up for this in their breath of application, ease of understanding, costeffectiveness of production, and potential to be used for benchmarking between localities. Furthermore, such indicators may also contribute to strategic information and policy decisions over a range of issues, such as guiding decisions on where to create new protected areas, and understanding the relationship between local provision of CES and the current demand for them between different localities.

Given these arguments, our objectives in this study are therefore to provide a summary and analysis of a range of potentially useful CES indicators which can be readily and costeffectively produced at a range of scales, taking the UK as an example. We also examine the degree to which these measures match what is required in a good indicator. 
2 In the following section we describe a set of indicators which can be produced at the level of 3 a single country (England), for Local Authority Districts (LADs) within a single nation state 4 (the UK), and to benchmark electoral wards and open spaces within LADs in England, using 5 The City of Nottingham LAD, located in the English Midlands, as an example. We produced 6 the indicators for Nottingham using data which are either currently available across the 7 whole of England, or which might be expected to be made so available in the future. In 8 producing these indicators we recognise a distinction between supply, i.e. the availability of opportunities for Cultural Practices within Environmental Spaces, and demand for CES, i.e. the needs, desires and intentions of people to exploit these opportunities. We do this within the NEAFO framework described above, measuring CES in terms of supply of, and demand for, Environmental Spaces and the Cultural Practices.

\subsection{Supply Side Indicators}

Few, if any, attempts have been made to provide a set of indicators which measure how the availability, or supply, of environmental spaces varies across an entire country. This is partly due to a lack of data on the location of such spaces, and how the benefits they deliver may vary from location to location. However, many distinct types of environmental space have been mapped comprehensively across all of the UK, all of Great Britain (i.e. England, Scotland and Wales, but not Northern Ireland), or across one of these four devolved nations.

2.1.1 Supply-side - Percentage cover or density measures of environmental spaces by local authority district

The NEAFO study was able to measure the percentage cover or density per unit area of 17 types of environmental space within Local Authority Districts (LADs) within Great Britain, using data which was publicly available or could be acquired cheaply under licence (a description of the data sets and processing used for all the indicators described in this study can be found in Church et al., 2014, pp 91-100). These data allowed mapping of percentage cover across all LADs, which enabled patterns of low or high provision to be discerned. The same base data sets used to calculate these indicators could be employed to calculate percentage cover in a radius around small geographical units (e.g. UK census output areas approx. 130 households) and could therefore be used for benchmarking at finer spatial resolutions. 


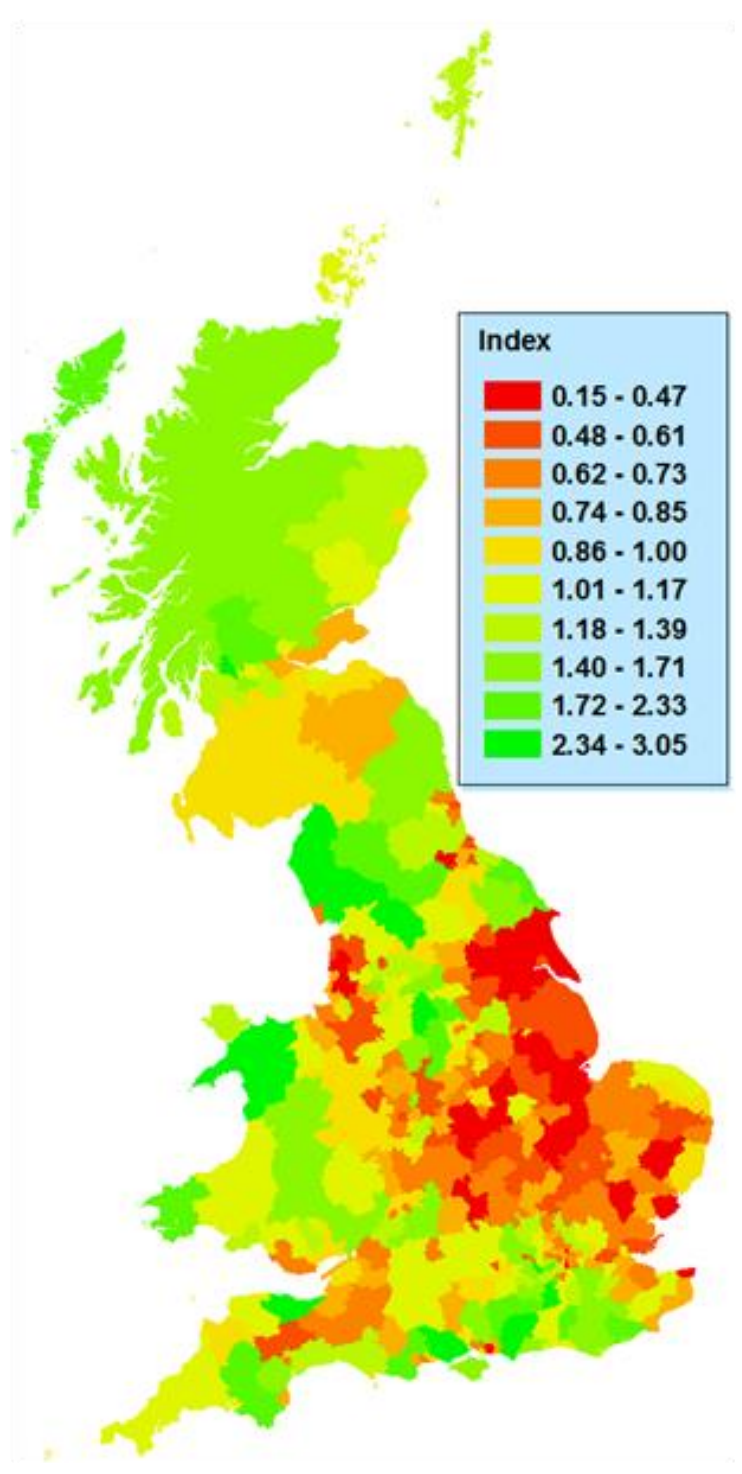

Figure 1. Summary Index based on 17 indicators for GB Local Authority Districts. Indicators included:

Average Garden Area per Household;

Number of National Trust (NT) Properties per Capita;

Percentage cover of the following types:

Non-urban Land; Country Park; National Park; Ancient Woodland; Broadleaved Woodland; Total Woodland; Sports \& Leisure Areas; Urban Green Space; Parkland; Mountain, Moorland or Grassland; Fresh Water; Garden; Land Designated for Conservation (excludes national parks); Open Access Land; NT properties.

Figure 1 shows the average value for all 17 indicators by LAD expressed as an index, and reveals that central and eastern areas of England display particularly low values for this index, and that LADs in northern Scotland, Wales and parts of northern and southern England display higher than average values. We found that there was relatively little redundancy across the different indicators, with few correlations between them either at below -0.5 or above 0.5 .

Mapping of this sort, although it reveals relatively little information about the quality and importance of specific environmental spaces at a local level, does allow benchmarking of localities against each other, and can therefore help decision makers to determine how provision of CES differs between them. We illustrate this in Figure 2, which shows how the Nottingham City Council compares to other LADs in Great Britain $(N=380)$, in England ( $N=$ 326) and to the 4 LADs which share a border with it. The data shows the authority's urban character, with a relatively high provision of Parkland, overall Urban Green Space, Sports and Recreation Facilities, and Country Parks and Gardens. For all these indicators, the LAD also has higher provision than the more rural authorities bordering it, England, and Great 
Britain as a whole. In contrast, there are no National Trust properties within its borders, nor National Park or Open Access Countryside. Woodland, land cover by grassland, mountain and moorland, and arable land is relatively scarce. Although relatively little of the authority consists of areas designated for conservation (such as SSSIs or Sites of Outstanding Natural Beauty) a relatively high proportion of the Authority consists of Nature Reserves, and the authority also has a relatively high percentage of open fresh water.

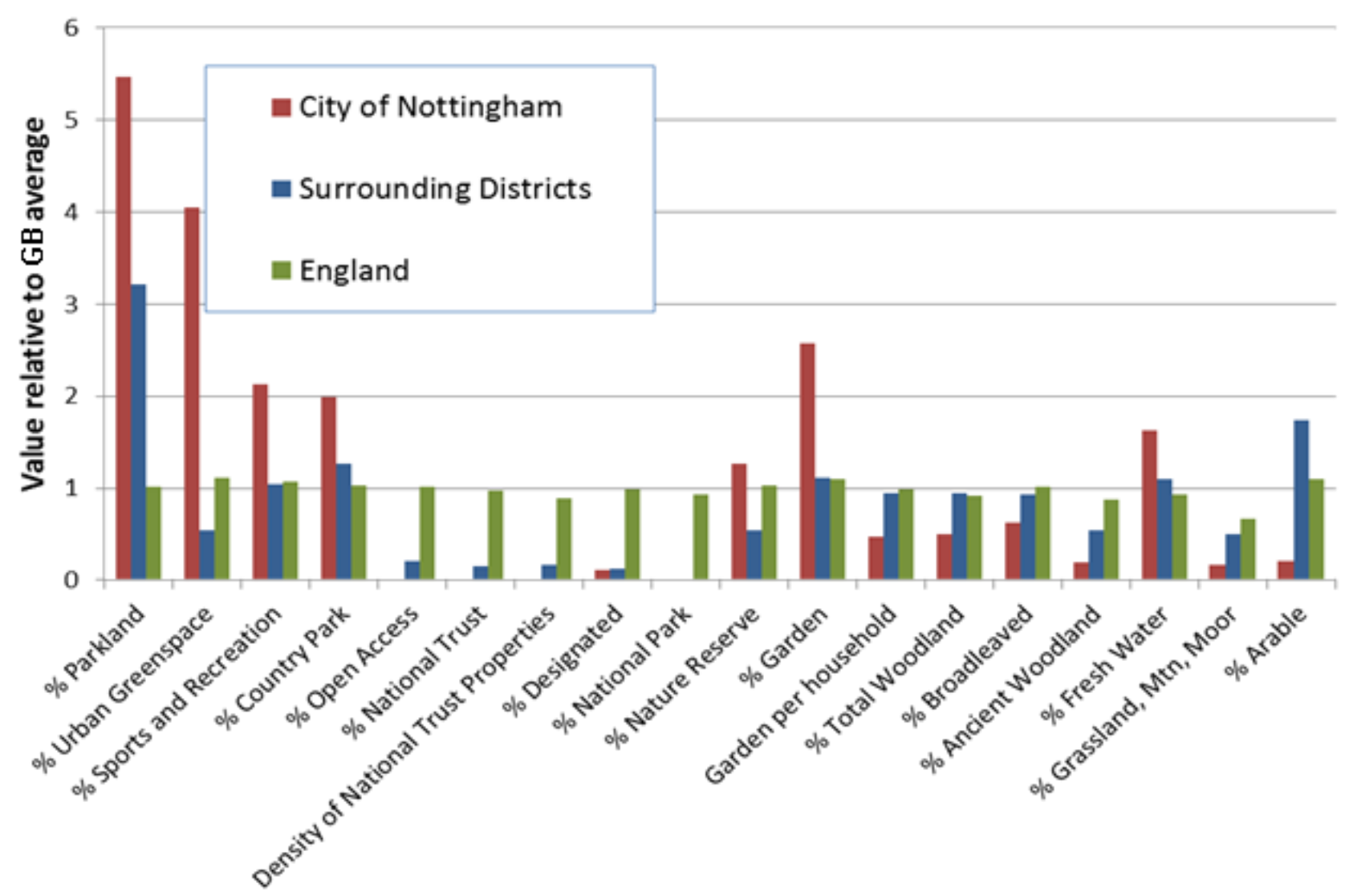

Figure 2. Indicators of stock of CES, relative to the average for all Local Authority Districts (LADs) in the UK, shown for England and the City of Nottingham LAD, and summarised for the 4 LADs bordering the City of Nottingham.

\subsubsection{Supply-side - Measures of access to environmental spaces, by local authority district}

Although indicators for the availability of environmental spaces allow benchmarking of the provision of CES, they do not reveal the degree to which these spaces can be accessed by those who would wish to visit them. To do that, we need to measure which of these spaces are publicly accessible as many environmental spaces are privately owned and public access to these is prohibited or restricted. We also need to measure how far, and by what means, people need to travel to access them, because the benefits they provide will differ according to how many people are able to visit them, although clearly some benefits may be derived by seeing places from afar, or simply knowing that they exist. In NEAFO, we addressed the first, access-related, issue by looking at two types of environmental space (country parks 
and nature reserves) where access is always provided to the public, and a further two, Ancient Woodland and Areas of Natural Vegetation, where access would often, but not always, be achievable via the UK's extensive Public Rights of Way network. We addressed the second issue by measuring the distance between households and these four types of environmental space. In calculating indicators from these data, we developed measures based on the UK ANGSt programme (Handley et al., 2003) to select sizes of space and distance from households to them. This enabled us to calculate a range of indicators for: i) the mean distance between households and the nearest example of 4 different sizes of environmental space $-2,20,100$ and 500 ha (see Figure 3); and, ii) the proportion of households within an area (we chose LADs) within a 300m, $2 \mathrm{~km}, 5 \mathrm{~km}$ and $10 \mathrm{~km}$, respectively of the nearest spaces of these different sizes, which match the distance/ size criteria used by ANGSt. Using socio-demographic data from the UK census, indicators of this sort can be readily calculated for groups of special interest to policy makers, such as children, the elderly, those living in deprived areas or with poor health outcomes.

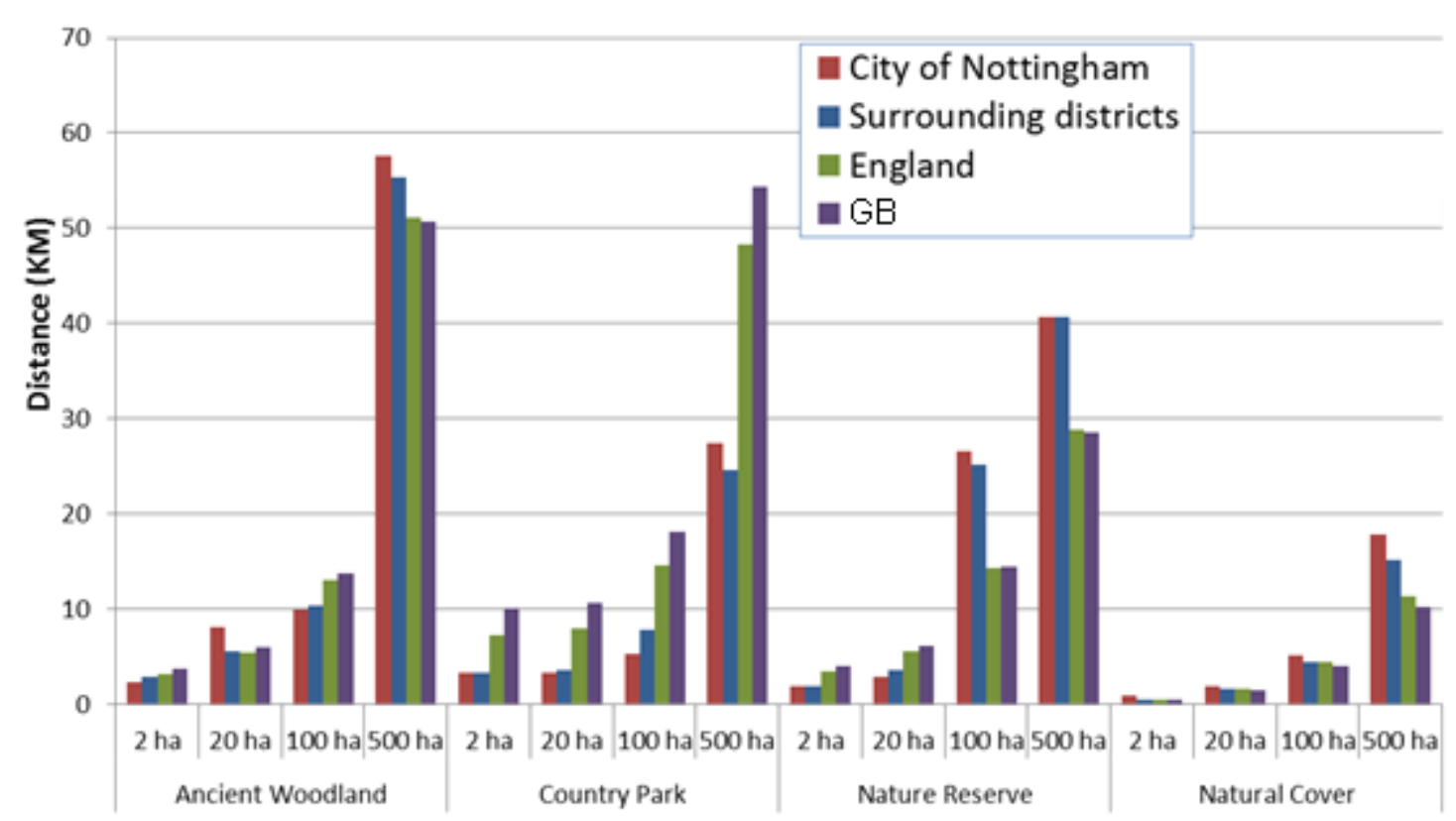

Figure 3. Average distance per resident to patches of 2, 20, 100 and 500 ha of Ancient Woodland, Country Parks, Nature Reserves and Natural Habitats (excludes Urban Environments, Agricultural/ Horticultural Land, Improved Grassland and Coastal and Fresh Water Environments). Data shown for England, City of Nottingham LAD and summarised for the 4 LADs bordering the City of Nottingham.

Figure 3 shows a mixed picture; in relation to the indicators for Ancient Woodland, Country Parks and Nature Reserves, Nottingham performed better than average compared to its peers in at least one size category and worse than its peers in at least one other. Only in the case of 'natural cover' were residents in this LAD worse off for all size categories. 
1 In the case of Nature Reserves, Figure 3 shows that a relatively large proportion of the Land

2 Area of Nottingham is taken up by Nature Reserves but that, in contrast, for larger reserves

3 ( $>=100$ ha) Nottingham residents are, on average, further away from such sites than any of

4 their comparator groups. This is explained by the fact that Nottingham is a relatively small

5 LAD but contains several Nature Reserves; however, none of these are over 100 ha in size.

6 Representing these data with reference to the proportion of the human resident population

7 within the respective ANGSt size/ distance criteria gave similar results.

\section{$9 \quad$ 2.1.3 Supply-side - Case study for electoral wards in the City of Nottingham LAD}

The supply related data we have illustrated so far has used data which is available for at least the whole of England. However, in the UK, many local data sets are held by local authorities but are not widely available. For NEAFO, we were able to obtain data for Nottingham City LAD for Tree Preservation Orders (TPOs), and the location of definitive Public Rights of Way (PROWs), neither of which are available as national data sets. We combined these data with other data available for the whole of Great Britain to calculate a set of indicators for the City of Nottingham's electoral wards ( $N=20$, with resident population sizes in 2011 of between 9,952 and 21,414 ). We used the PROW maps, as well as UK Ordnance Survey mapping of minor roads and urban paths, to calculate the total length of these features which passed through natural environments (all habitat types excluding built up areas and gardens) within a 1 mile $(1.6 \mathrm{~km})$ radius of each ward. We used the TPO data as a proxy for the presence of mature trees of cultural significance within the ward.

Other ward-level indicators measured the average area of garden per household and the percentage cover of a variety of land use/land cover types. Of these, Vegetated Land consisted of all areas classified by the UK Ordnance Survey as not man-made or gardens, including very small areas such as road verges; Natural Cover consisted of all habitats not classified by a UK-wide land-cover map as urban areas and gardens or as agriculture or horticulture (minimum mapping unit: 0.5 ha). 

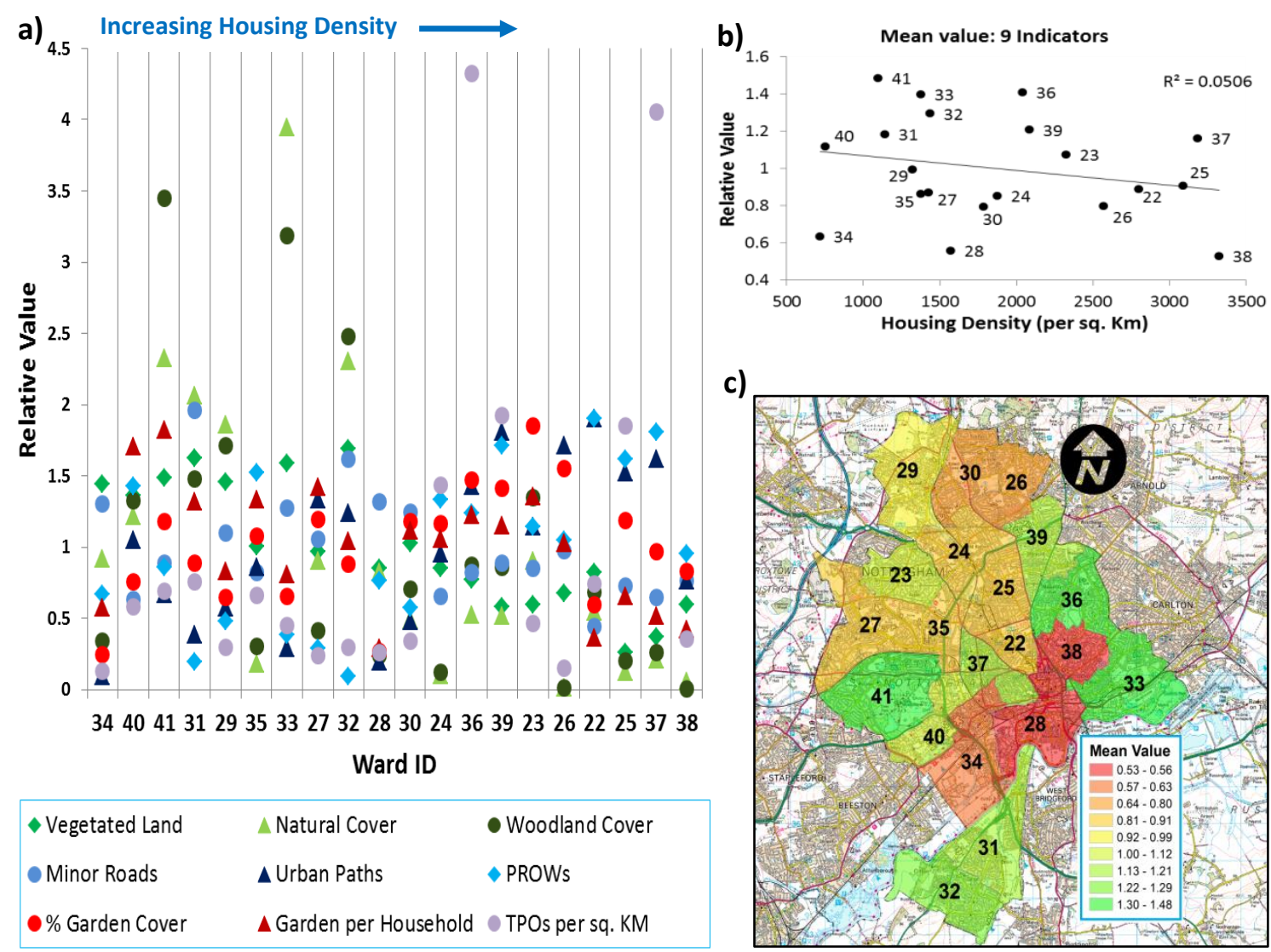

Figure 4. a) Nine CES indicators for electoral wards in the City of Nottingham Local Authority District, each indicator shown relative to the mean value for all wards. Wards are ordered, left to right, by housing density; b) the average value for all nine indicators plotted against housing density for each ward (label for each point show Ward IDs from figure 5a) and c) a map of the values shown in 5b, with labels showing Ward IDs.

The information in Figure $4 a$ and $b$ reveals a high degree of variability, both between wards and between indicators for a given ward, although there was a weak negative relationship between housing density and average values aggregated over all indicators. Mapping these data (Figure 4c) revealed that values were lowest amongst wards around the city centre (28, 34 \& 38), lowest in some of the outlying wards in the South, West and East of the LAD and generally at intermediate levels in the northern half of the authority.

2.1.4 Supply-side - Case study for the quality of environmental spaces in the City of Nottingham LAD

As we discuss above, the quality of environmental spaces is likely to differ from location to location. van Herzele \& Wiedemann (2003) found that the contribution that a given environmental space makes to human wellbeing is likely to differ with regard to the following factors: space size, contact with nature, quietness, safety, availability of facilities, and culture and history. Although other studies have suggested additional factors which may contribute towards the quality of environmental space, e.g. landscape heterogeneity (Plieninger et al., 2013, Casado-Arzuaga et al., 2013), 'beauty' (Plieninger et al., 2013), tidiness (Morgan, 1990) and, in the case of blue spaces, water quality (Morgan, 1990), we used Van Herzele \& Wiedemann (2003) as a starting point for incorporating quality 
measures into CES indicators, as this study was conducted on urban parks, which are: i) arguably one of the most important spaces for local inhabitants; and, ii) are a highly managed and 'human' environment, and so the interplay of culture and nature are particularly important.

We again used the City of Nottingham LAD as a case study area, and were able to obtain information, from a variety of national and local data sets with which to create indicators for all outdoor green spaces in the authority. In total we created 10 indicators. To map environmental spaces we were able to draw on a GIS layer of open spaces provided by Nottingham City Council, which classified open spaces as: i) Amenity Green Space; ii) Cemeteries and disused churchyards; iii) Agricultural land; iv) Allotments, community gardens and urban farms; v) Outdoor sports facilities; vi) Natural and semi-natural areas; vii) Institutional; viii) Provision for children and young people, and ix) Parks and gardens.
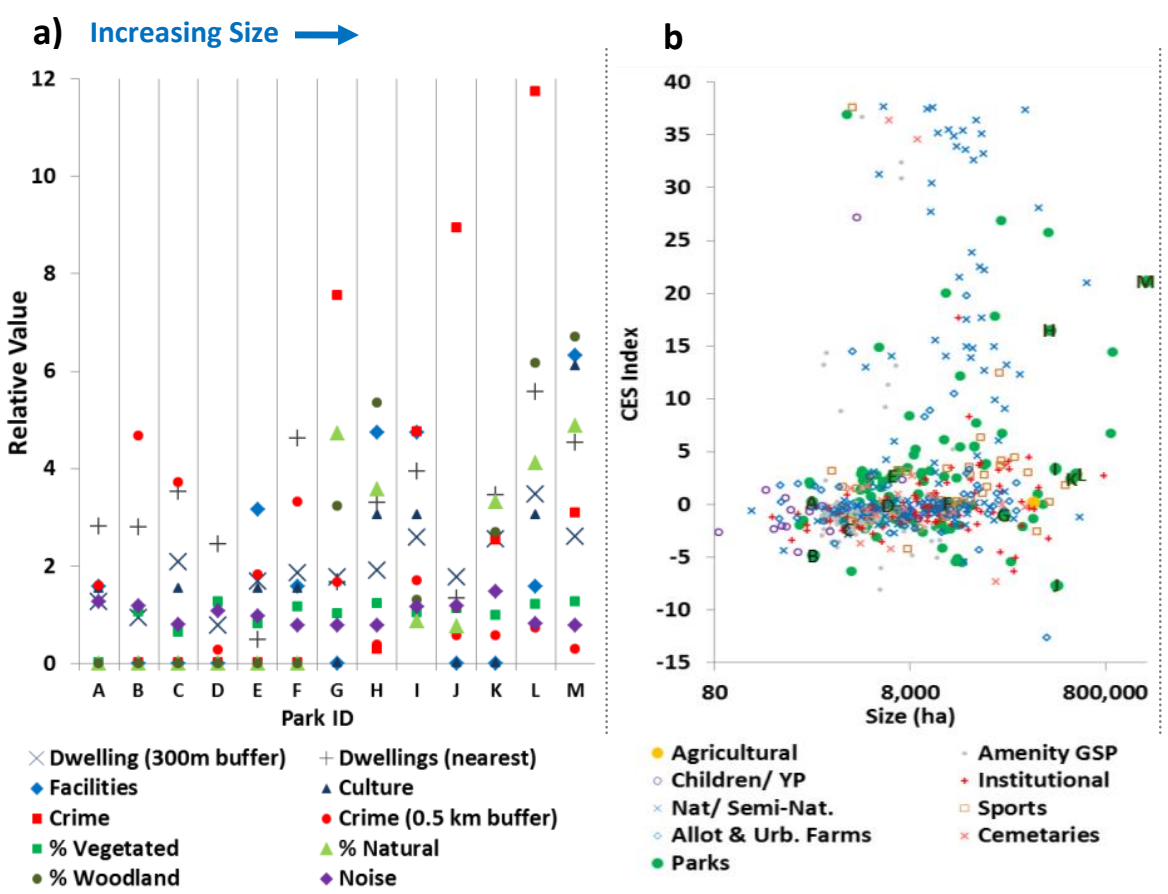

c)

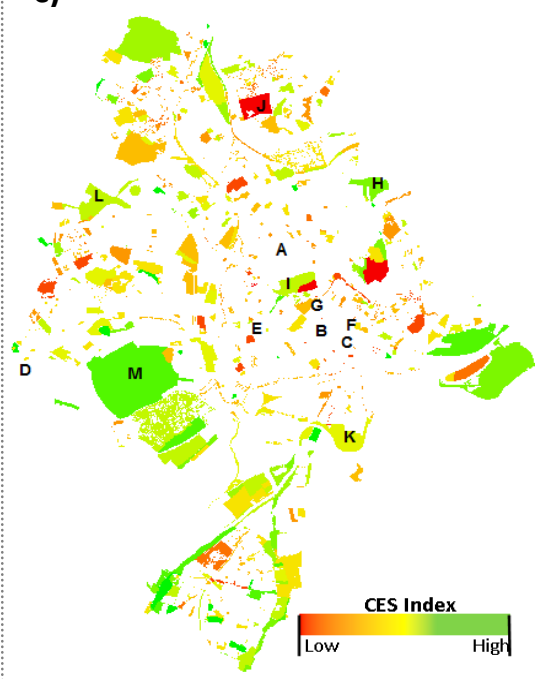

Figure 5. a) Ten CES indicators for 13 parks in the City of Nottingham Local Authority District. The value for each indicator is shown relative to the mean value of this indicator for all parks in the LAD. The sequence of parks shown, left to right, is in order of increasing size. A value of 1 represents the average value for all parks; b) A CES index based upon the values showing in $\mathbf{5 a}$ (excluding the two indicators based on the number of dwellings close to the open space) for all open spaces in The City of Nottingham LAD, plotted against the size of the park. Data points are shown for each of 648 open spaces in the LAD and grouped according to open space type. The 13 parks examined in Figure 5a are labelled; c) A map of the CES index for each open space within the LAD, with the same labels used for 13 parks.

Two of these indicators measured the likely number of potential users of green spaces, namely the number of residential addresses (dwellings) within a $300 \mathrm{~m}$ buffer of each space, 
and the number of dwellings within the LAD which were closer to the space than they were to any of the other spaces. A further two indicators used the number of text labels on UK Ordnance Survey maps referencing heritage features and facilities; the positioning of the text over each open space was used as proxies for the quality of facilities and the strength of association between each space and local culture and heritage. Average noise levels were estimated using preliminary modelled data produced for the City of Nottingham by the UK Department for Rural Affairs (DEFRA).

Two proxies for safety were used, both of them calculated from mapping of reported crime by the UK Police Force, May-August, 2013: i) the number of crimes within each open space; and, ii) the number of crimes per $\mathrm{km}^{2}$ within a $0.5 \mathrm{~km}$ radius of the open space. We used this latter data both because crimes committed within open spaces may be geo-referenced to nearby private addresses, and on the assumption that high levels of crime in the surrounding neighbourhood contribute to a sense of danger in public spaces. These data excluded crime types such as burglary and fraud, which are typically committed indoors. We were unable to obtain data for perceived levels of safety.

We calculated three indicators based on land cover, using some of same base data sets used for the ward-level indicators: \% Vegetated, \% Natural and \% Woodland. As an example of how these data could be used to examine the distribution of CES across Urban Parks and other environmental spaces, we selected those parks ranked within the top ten for either of the two indicators related to the number of potential users, which resulted in a list of 13 parks. We then calculated the value, relative to all open space within the LAD, for each indicator, and plotted these, ordering them according to the size of the park, which varied from $791 \mathrm{~m}^{2}$ to 207 ha (Figure 5a).

In a similar manner to the ward level analysis described earlier, this analysis revealed a high degree of variability between the 13 parks and a high degree of variability between indicators for a given park (Figure 5a).

We also computed a simple aggregated index from these data, using the formula: CES Index $=$ Facilities + Heritage + Average $(\%$ Vegetated $+\%$ Natural $+\%$ Woodland $)-$ Noise $-($ Crime within the park + Crime (within a $300 \mathrm{~m}$ buffer). We plotted the value of this index against size of the open space, for all open space types (Figure 5b) and also mapped these data (Figure 5c). Both figures revealed a high degree of variability across parks in the CES index, showing that provision of CES varied widely between localities and different types of open space.

\subsection{Demand Side Indicators}

In contrast to the supply of CES, which is determined largely by the location and quality of individual environmental spaces, demand is determined largely by the preferences, values and practices of local populations. This must be estimated either by observing human behaviour, such as visitor rates or spending related to enjoying CES, or through eliciting the information through questionnaires or other consultation exercises. Although numerous local-level studies have used these techniques (e.g. Plieninger et al., 2013, Cazado-Arzuaga 
et al., 2013, Fagerholm et al., 2009), there are currently few data sources of this type which are sufficiently large or comprehensive to produce CES indicators across an entire country at a similar level of spatial desegregation to the supply side indicators for LADs described earlier. In most cases these public-engagement methods are too resource intensive to sample the entire population of a country adequately and therefore cannot be used in national-level benchmarking. Possible exceptions might include data available for specific activities, such as membership of angling groups, or for specific environmental spaces, such as visitor rates at local nature reserves.

Within the UK, the largest and most comprehensive survey that could be used to measure demand for CES is the Monitor of Engagement with the Natural Environment Survey (MENE) (Natural England, 2011 a, b). This is an on-going, face-to-face and in-home questionnaire conducted across England, which now includes over 57,000 spatially referenced interviews over a four year period. Questions are asked about a range of public environmental spaces (e.g. woodland, farmland, mountain, river, country park, city park, allotment, beach, etc.) and a number of cultural practices in these (e.g. enjoying peace and quiet, relaxing and unwinding, spending time with family and friends, entertaining children, exercising and improving health, exercising the dog, enjoying scenery, and watching wildlife). As such, MENE has the potential to act as the basis for a range of indicators relating to demand for CES, aggregated at the national level. MENE can be used to measure the proportion of the population who visit environmental spaces of different types, the activities in which they engage in each of these spaces, their expenditure, travel costs and the subjective well-being they experience in being there. These measures can in turn be used to monitor change over time at a national level.

MENE also contains a great deal of socio-demographic data on those who take part in the survey. Indicators can therefore be created not only for the population as a whole, but also for different socio-demographic classes - an example which would appear to be of clear use to policy makers would be the proportion of children living in socially deprived areas who visit environmental spaces within a specific time period. However, although the sample size for MENE is large, it is still inadequate for measuring demand for specific cultural practices or environmental places at finer resolutions than a regional level. Nevertheless, for this study we were able to use the socio-demographic characteristics of respondents to MENE (such as age, sex, social grade, marital status, whether they had children, a car etc.) and their preferences for certain types of spaces and activities within each type of environmental space, against data from the UK 2011 Census, from which many of the same socio-demographic characteristics used in the MENE survey can be mapped at fine spatial scales for almost the entire UK population (Office for National Statistics, 2013). Doing this, we were able to calculate the probability that a given individual would visit one of the MENE classes of environmental space in a given week, and engage in any one of the various types of activity described in MENE.

It should be acknowledged that such mapping methods assume that these relationships play out in a consistent manner throughout the area covered by the census, and do not take into account local-level effects which might be important in the understanding of how true levels of demand for CES differ between localities. However, the strength of using such modelling techniques to estimate local demand is that, combined with information on the supply of 
environmental spaces, they can be used to estimate the degree to which local supply matches local demand, and can therefore provide insights to decision makers on where more effort needs to be made to ensure an improved supply of CES to local populations. An example of this is shown in Figure 6. To produce this figure, mean values were calculated for the four supply-side accessibility indicators relating to the proportion of the adult population within the relevant ANGSt distance/ size criteria from country parks, described earlier in the section on "Supply-side - Measures of access to natural spaces, by local authority district". This value was then divided by the probabilities we calculated from the MENE data for visiting country parks. This resulted in a proxy measure of the degree to which supply matches demand for visiting country parks. It can be seen from the map that many districts in eastern and northern England and in the West Midlands had low scores for this indicator, suggesting that supply for country parks may struggle to match potential demand in these areas.

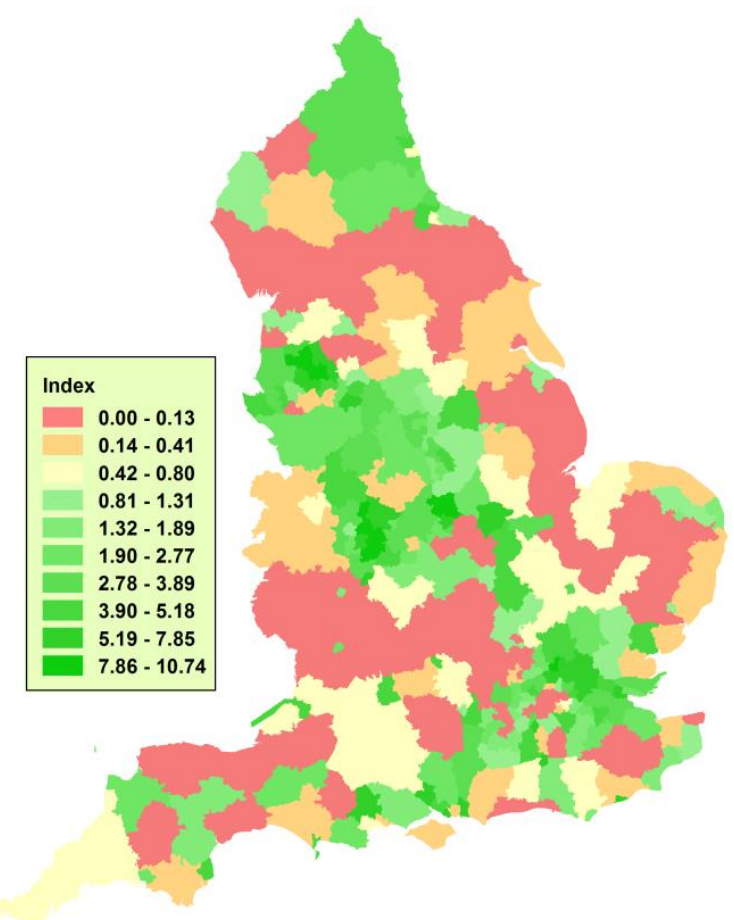

15
Figure 6. An index of the relationship between supply and demand for Country Parks, shown for all English Local Authority Districts, and calculated as follows:

A. The probability of visiting a country park within a given week was calculated on the basis of the socio-demographic profile of each district and preferences for specific types of cultural practice and environmental place, as revealed in responses to the MENE questionaire conducted accross the whole of England.

B. For each district, the mean proportion of the population living within each of four distance/size of open space criteria was calculated for country parks.

B/A was calculated as a proxy for the relationship between supply and demand. $B / A$ is shown as an index in the figure. 


\subsection{Evaluation of CES indicators}

Having described some of the difficulties entailed in calculating cost-effective indicators for CES across various scales, and presented approaches to addressing some of these through the creation of indicators from publicly available data, we will examine the degree to which they match criteria for successful environmental indicators

We have presented a range of indicators for measuring CES, chosen primarily on the basis of their cost-effectiveness, in that they were all derived from data that is already publically available or can be readily obtained from third parties at little or no cost. Although in some cases a considerable amount of data processing was required to produce the indicators, this could be automated so that, once the data had been assembled, relatively little effort would be required to reproduce or update them for any geography within Great Britain, or at least, England alone. This of course assumes that the primary data, which had been produced, often requiring considerable resources, for purposes other than the creation of indicators, will continue to be available.

However, to count as suitable indicators these measures clearly need to fulfil criteria other than how expensive or time consuming they are to produce. Environmental indicators, as opposed by those specifically designed to measure CES, are long established and various criteria have been used to evaluate them, with a literature extending back over 20 years or so (Environmental Challenge Group, 1995, Anderson, 1991, CSIRO 1998, Audit Commission, 2005). From this literature, we have identified the following broad set of criteria against which to evaluate our CES indicators.

Criterion 1. Indicators should be meaningful and represent something believed to be important in its own right. Our GB-wide and ward level supply side indicators measure the availability of certain types of environmental space or the habitats associated with them. All of these spaces are enjoyed by people for a range of CES related benefits, such as relaxation, exercise, a feeling of being at one with nature and inspiration. Indicators which measure proximity to these features additionally measure the degree to which some of these benefits can be realised by different sectors of the population. Finally, the indicators we calculate for open spaces in Nottingham aim to identify the degree to which the quality of environmental spaces varies locally, which will affect the benefits that can be derived from them, whether through affecting the experiences of those accessing those spaces or through the degree to which these factors encourage them to access, or discourage them from accessing, these spaces in the first place.

Similarly, demand-side indicators based on MENE data aim to measure how important these environmental spaces are to people and additionally what practices are most popular in them. Indices based on relationships between our LAD-level supply and demand-side indicators can be used to identify the degree to which supply matches demand.

Different indicators are likely to be found useful by different groups - for example, planning departments might use access to country parks and other public spaces as a way of identifying areas where there should be more provision, conservation groups might use 
information of poor access to and stock of habitat types such as ancient woodland as an evidence base for preservation, and local parks departments might use information on the quality and attractiveness of local parks as a way of indentifying where improvements need to be made.

Criterion 2. Indicators should have an agreed and scientifically sound meaning which can be readily understood by and communicated to the target audience. For the most part, our indicators have a clear scientific meaning, in that they measure the amount of something, the distance to something, or the degree of something (such as noise). However, further work is required to better understand the relationships between these quantitative measures and human benefit.

For example, our comparison of the City of Nottingham with other Local Authorities found that the population of this authority were relatively well served with small nature reserves (<= 20 ha. ) but less so with large ones ( $>=100$ ha.). It might seem obvious that small reserves and large reserves are not perfect substitutes, that people are likely to prefer to visit large rather than small ones, and that the importance of size is likely to vary by location with reference to factors such the people using it, the activies conducted there and the habitat requirements of flora and fauna. However, little research has been conducted on how and under what circumstances benefits vary with size.

\section{Criterion 3. Indicators should have a sound and practical measurement process resulting in the creation of quantitative measures (even when based on qualitative data). All the indicators presented in this study consist of quantitative measures, usually produced by national or local government agencies using an agreed methodology. Some of the habitat measures were derived from interpretation of satellite imagery used in the 2007 CEH Land Cover Map, which may be subject to errors in interpretation. Similarly, the calculation of noise bands in urban green spaces uses modelling methodologies which are still being developed and refined. Finally, in some cases we have used spatial cut-off points which seemed sensible to us but not based on known relationships (for example, the use of a one mile buffer around ward boundaries to capture the area within which residents are most likely to want to use footpaths and roads for walking).}

Criterion 4. There should be only a short time-lag between the state of affairs referred to and the indicator becoming available. Our supply-side indicators are based on data that are updated irregularly. However, once the these data are made available the indicators can be calculated rapidly, and in any case changes in CES caused by changes in the configuration of environmental spaces are often felt as broad patterns which are subject to only gradual change. Our demand side indicators derived from MENE are updated regularly, although adequate sample sizes for indicators need to be acquired over relatively long periods. Some of our data use national census data which are only updated at ten yearly periods.

Criterion 5. Indicators should have the potential to monitor change and assess and evaluate progress over time. One clear advantage of all the indicators we describe is that they can be used to assess change over time, and this is limited only by how often the base data they are calculated from are updated. In the case of some data sets, there is a need to 
ensure that the base data are acquired using the same techniques and methodology when they are updated. For example, the indicators based on habitat cover come from the 2007 UK Land Cover Map, which does not use the same methodology as its 2000 predecessor, making temporal comparisons problematic.

\section{Criterion 6. Indicators should facilitate comparisons of performance between different geographical areas, ideally including international comparisons. A particular strength of our indicators for GB LADs is that they can be disaggregated to any geography within Great Britain. Furthermore, initiatives to standardise and share local authority level data, such as the UK INSPIRE programme ${ }^{1}$ mean that some of the indicators we are currently able to produce at local scales (TPOs, PROWs, mapping of open spaces) may shortly be available in a standardised format over much larger areas. Many of our LAD-level supply-side indicators could be adapted to settings outside the UK, especially those measuring the availability of habitat types or the location of designated sites such as nature reserves and national parks with an agreed international meaning.}

Our ward level and local parks indicators for Nottingham can be used to identify those areas within a local authority where provision of CES is better or worse, and therefore to act as an evidence base for decisions about what aspects might need to be fostered or improved. These indicators can be either aggregated as a single measure of overall quality (see, for example, Figs. $4 b, 4 c, 5 b$ and $5 c$ ) or disaggregated to examine the degree to which different mesures vary between each area (see, for example, Figs. $4 a$ and $5 a$ )

Although the indicators we present were developed for a UK context, many of them could be used for benchmarking different countries against one another, or could be adapted for settings outside the U.K., especially those measuring the availability of habitat types, such as woodland, or the location of designated sites such as nature reserves and national parks with an agreed international meaning.

Criterion 7. An indicator should serve a useful purpose, e.g. it can used to inform improvements in policy or the better management of resources, or help focus information to answer important questions or to help review, justify and set local objectives and priorities. This is clearly a key component of any indicator. All the indicators we describe could be called 'generic', in that they were not designed to address a specific management question but rather to provide top-level information on the supply of and demand for a range of environmental spaces and cultural practices. However, the issues they address are important and of policy relevance. Furthermore, they could be used to address specific management issues. Examples of these include the following: evaluation of the CES impacted by agri-environment schemes; identification of areas where CES could be improved by the creation of new designations areas for conservation or recreation areas, such as National Parks, Nature Reserves, Nature Improvement Areas and Country Parks; identification of areas where the designation of new PROWs, such as encouraged by the UK's Paths for Communities Programme ${ }^{2}$ would increase access to CES; identification of

\footnotetext{
${ }^{1}$ http://data.gov.uk/location/inspire

${ }^{2}$ http://www.naturalengland.org.uk/ourwork/access/rightsofway/p4c.asp
} 
areas where planning constraints should be introduced to protect key CES resources, where provision of CES is currently poor, or in areas of high deprivation. The indicators we describe can be used to understand how supply of CES varies locally and how demand is likely to vary with this, and they can therefore be used to set priorities for the protection and enhancement of these services.

Criterion 8. Indicators should draw on information which is readily available, and assist decision making by being effective and cost-efficient to use. All of the indicators we used were based on information which was readily available at no or little cost.

Criterion 9. Indicators should stimulate debate and raise awareness. The indicators which we describe are mainly used to monitor change in provision of or demand for CES. The ability to map the data means that differences across the UK or within a local area can be easily discerned, which we believe stimulates debate about the provision of CES and how this needs to be take account of.

\section{Discussion}

We have presented a range of indicators which can readily calculated at various scales and used to meaure variability in provision of CES across time and space. By arming decision makers with measures by which they can benchmark provision of CES across space and time, we can give them an evidence base to better evaluate where provison needs most urgently to be improved. However, the indicators and analyses we present have not been produced so as to address any specific management question but rather to illustrate what CES indicators can be produced using currently available data. It is hoped that our work will stimulate debate and further research towards arriving at a agreed set of indicators which can be used to inform both policy and management decisions. In the remainder of this discussion we will address potential criticisms of the approach we have adopted and discuss the research issues which we think need most urgently to be addressed if we are to arrive at a useful and widely adopted set of CES indicators.

\subsection{Possible criticisms of this approach}

CES are rich and multifaceted and therefore any set of indicators is likely to appear incomplete and only partly to measure the full range of services provided. There are some clear and obvious gaps in the indicators we present. Two key components are missing from our supply-side indicators due to lack of data. One is information on the quality of individual environmental spaces, although our local level study of open spaces in Nottingham provides some insights into the way that issues of quality can be addressed. The other is in mapping public access. Although spaces such as Nature Reserves and Country Parks are almost always accessible to the public, others such as ancient woodland and fresh water environments are often on private land with restricted access. Thus we recommend that public access to such areas is mapped so that accessibility measures can be calculated taking this information into account. Mapping of entrances to public open spaces would also allow 
more sophisticated approaches to calculation of travel distances via road and path networks (Barbosa et al, 2007).

We have not addressed indicators for the availability or quality of marine environments in this study, although they are clearly important in delivery of CES in island nations such as the UK. Information on access to the coast, and specifically sandy beaches, as well as indicators of quality such as water quality, the cleanliness of beaches and the facilities available on them could be used to construct indicators of this sort.

Our demand-side indicators are less well-developed than those on the supply side. This is not because we believe they are less important but rather because they is currently relatively little suitable information related to the demand for CES, particularly of the sort which can be matched to supply. We have given one example of a demand-related indicator - the proportion of the population likely to visit country parks. We used this example because we were able to compare data for country parks on both the supply and demand side. However, in most cases such a matching is not available, and this makes calculation of a suitable index of demand versus supply problematic. It might be argued that many environmental spaces may act as substitutes for one another and a composite index might be desirable, matching demand for all or a selection of environmental spaces represented in the MENE survey with data on supply of the same type of spaces. However, this is stymied by the fact that in many cases there is no one to one relationship between the environmental places represented in MENE and data we have for the supply side. We have also made little attempt to link indicators of environmental quality to demand for CES. This is again because of a lack of suitable data, rather than because we believe that such indicators would not be useful.

Another possible criticism is that our approach appears weighted towards looking at CES as enjoyed primarily through recreational activities, as opposed to other ways in which these services are experienced, such as through inspiration, a feeling of belonging to a particular place, through spirituality, volunteering or educational activities. Our indicators often measuree access to, and the quality of, spaces which are suitable for recreational activities. However, we would argue, firstly, that recreational activities form an important part of the way in which the benefits of CES are realised and that, secondly, these other benefits, which might be distinguished from recreation, are for the most part mainly engendered through direct experience of environmental spaces, and that it is therefore important to be able to evaluate access to such spaces if these other benefits are to be fully understood. Furthermore, many of our indicators could be used as proxies for CES which are not related specifically to access or recreation - for example, \% cover of ancient woodland, nature reserves and so on might be used to estimate potential habitat for wildlife valued as a CES in its own right.

Our place-centred approach may be seen also be seen as a rejection of the identifaction of CES with specific characteristics of the environment, an approach typically used in the identification of other ecosystem services (see, example, in Bieling et al., 2014, for an application of this approach to CES). However, our approach sees such characteristics as contributing to the quality of CES provided by each environmental place rather than CES in themselves. For example, our indicators for particular types of woodland (e.g. ancient, 
broadleaved), acknowledge that the quality of the CES offered by an environmental space may change according to the composition of the ecological communities in them.

Heritage values are recognized as a key component of CES and are one of the six components of CES recognized by the Millennium Ecosystem Assessment (MA, 2005). We attempted to address this issue through one of the 'culture' quality measure we used for green spaces in Nottingham, our indicators based on National Trust Sites, and also in NEAFO explored the degree to which the 'heritage and antiquities' text labels within Ordnance Survey MasterMap ${ }^{3}$ data $(N=90,841)$ could be used as a proxy measure of the heritagerelated significance of environmental spaces (Church et al., 2014). However, the indicators presented here do not address CES related to heritage in any depth. The way in which ecosytems relate to heritage can only be fully understood through an in-depth understanding of the historical and cultural associations of a given location (Tenberg et al., 2012), and more research is needed on whether proxy measures can be developed at broad scales, using existing data as indicators for CES related to heritage.

Another possible criticism of our approach is that, in endorsing the environmental place/ cultural practice framework used by the NEA, it fails to address certain CES which do not fit neatly within this framework. For example, many highly mobile species such as migrant birds (the barn swallow Hirundo rustica and common cuckoo Cuculus canorus are examples) have a strong cultural resonance, but are not strongly associated either with a set of environmental spaces or cultural practices. Some cultural practices such as artistic expression may be inspired by a range of environmental spaces or the biota which are found in them, but are not be linked to any specific type. Similarly, enjoyment of CES through media such as magazines, documentaries or social media may only loosely be linked to specific environmental spaces. As we have made clear, these indicators specifically measure the stock of, proximity to, and demand for, certain types of open spaces and practices within them, and further research is needed to identify indicators which could be used to measure aspects of CES which do not fit neatly into these categories.

Some of our attempts to measure quality in enviromental spaces may appear to have little to do with ecosytem services (measures related to noise and safety, for example). However, we have included these amongst our indicators because they are likely to influence the benefits experienced by those enjoying CES, and therefore contribute to the quality of the CES delivered by a space. Information related to these factors are likely to influence decisions about how spaces delivering CES are managed, and it is therefore appropriate to incorporate these measures of quality into our indicators.

\subsection{The way forward}

Clearly, the indicators we present in this study can only act as proxies for the services provided by environmental spaces and the cultural practices undertaken in them. There is a need for more research work to identify the precise nature of the services (see Keniger et

\footnotetext{
${ }^{3}$ http://www.ordnancesurvey.co.uk/business-and-government/products/mastermap-products.html
} 
al., 2013), who the beneficiaries are (Satz et al, 2013), how different assemblages or environmental spaces may complement each other (Martin-Lopez et al., 2009), the role of aspects such as the environmental space size, landscape heterogeneity land cover patch size and accessibility, and aspects of quality such as environmental noise and soundscapes, aesthetic appeal (van Berkel \& Verbrug, 2012, Casalegno et al. 2013, Schirpke et al., 2013, Frank et al., 2013), biodiversity (Fuller et al., 2007, Dallimer et al., 2012) , safety and the culture and heritage aspects of environmental spaces (Tenberg et al. 2012).

Local priorities may dictate the need for indicators specific to single localities or a collection of localities; for example, Nature Improvement Areas (NIAs) in the UK allow for the development of area-specific indicators alongside those that can be compared between NIAs (Natural England, 2012). We would recommend that locally available data should be made accessible to a wider range of researchers and decision makers, as in envisaged by the UK's INSPIRE programme ${ }^{4}$. Particularly useful data sets in a UK would be the mapping of PROWs, which enable access to natural places in the countryside, and locally-held data on tree cover, which could be used to assess the attractiveness of urban environments, as well as the presence of tree-lined streets (one of 23 types of environmental place used in the MENE survey). The availability of a UK-wide PROW dataset would allow mappping accessiblity across the UK more accurately than is currently achievable - for example, the analysis of accessibiltiy to ancient woodland and other natural areas detailed in our study could be done so as to exclude those areas not accessible via road or PROW. Currently data on PROWs must be obtained from individual Local Councils, of which there are over 400 in the UK.

Numerous case studies illustrate how local perspectives can be taken into account in detailed, resource-intensive assessments of CES (see, for example, Balram et al., 2005, van Berkel \& Verburg, 2012, Schirpke et al, 2013, Nahuelhaul et al., 2013, Frank et al., 2013, Plieninger et al., 2013, Casado-Arzuaga et al., 2013, Casalegno et al., 2013, Klain et al.,2012). Whilst we do not question the validity and importance of these approaches as means of investigating and coming to a better understanding of CES, we believe the indicators we describe in this study constitute an important first step towards producing a set of standardised, cost-effective and informative indicators which can be rolled out across wide geographic areas. Using these standardised indicators different localities can be readily benchmarked against one another, or they can be used as a stepping-stone prior to the incorporation of local perspectives on supply and demand.

\section{Acknowledgements}

This work was carried out at the University of Nottingham and funded under the National Ecosystem Assessment Follow-on (NEAFO) programme- see- http://uknea.unepwcmc.org/Home/tabid/38/Default.aspx. We are grateful to two anonymous reviewers,

\footnotetext{
${ }^{4}$ http://data.gov.uk/location/inspire
} 
whose suggestions resulted in improvements to the manuscript, and to Lara Byrne for proof-reading.

\section{References}

Andersen, V. (1991). Alternative Economic Indicators. New Economics Foundation. London: Routledge.

Audit Commission (2005). Local quality of life indicators - supporting local communities to become sustainable. A guide to local monitoring to complement the indicators in the UK Government Sustainable Development Strategy. $32 \mathrm{pp}$.

http://collections.europarchive.org/tna/20080530153425/http://www.auditcommission.gov.uk/Products/NATIONAL-REPORT/0D488A03-8C16-46fb-A4547936FB5D5589/QofL2005.pdf

Balram, S. \& Dragícevíc, S. (2005). Attitudes toward urban green spaces: integrating questionnaire survey and collaborative GIS techniques to improve attitude measurements. Landscape and Urban Planning 71 147-162.

Barbosa, O., Tratalos, J.A. Armsworth, P.R., Davies, R.G., Fuller, R.A., Johnson, P. \& Gaston, K.J. (2007). Who benefits from access to green space? A case study from Sheffield, UK. Landscape and Urban Planning 83 (2-3) 187-195.

Bieling, C. \& Plieninger, T. (2013). Recording manifestations of cultural ecosystem services in the landscape. Landscape Research, doi: 10.1080/01426397.2012.691469

Brown, G. \& Raymond, C. (2007). The relationship between place attachment and landscape values: toward mapping place attachment. Applied Geography, 27, 89-111.

Casado-Arzuaga, I., Onaindia, M., Madariaga, I. \& Verburg, P.H. (2013). Mapping recreation and aesthetic value of ecosystems in the Bilbao Metropolitan Greenbelt (northern Spain) to support landscape planning. Landscape Ecology. doi 10.1007/s10980-013-9945-2.

Casalegno, S., Inger, R., DeSilvey, C. \& Gaston, K.J. (2013). Spatial Covariance between Aesthetic Value \& Other Ecosystem Services. PLOS ONE 8 (6): e68437. doi:10.1371/journal.pone.0068437.

Chan, K.M.A., Goldstein, J., Satterfield, T., Hannahs, N., Kikiloi, K., Naidoo, R., Vadeboncoeur, N. \& Woodside, U., (2011) Cultural services and non-use values. In: Kareiva, P., Tallis, H., Ricketts, T.H., Daily, G.C. \& Polasky, S. (eds.). Natural Capital: Theory and Practice of Mapping Ecosystem Services. University Press, Oxford, 206-228.

Chan, K.M.A., Satterfield, T. \& Goldstein, J. (2012). Rethinking ecosystem services to better address and navigate cultural values. Ecological Economics 74, 8-18. 
Church, A Burgess J and Ravenscroft N, Bird, W. Blackstock, K. Brady, E. Crang, M. Fish, R. Gruffudd, P. Mourato, S. Pretty, J. Tolia-Kelly, D. Turner, K. \& Winter, M. (2011). Cultural Services: UK National Ecosystem Assessment_Chapter 16 (UNEP-WCMC, Cambridge) 633-92.

Church, A., Fish, R., Haines-Young, R., Mourato, S., Tratalos, J., Stapleton, L., Willis, C., Coates, P., Gibbons, S., Leyshon, C., Potschin, M., Ravenscroft, N., Sanchis-Guarner, R., Winter, M. \& Kenter, J. (2014). UK National Ecosystem Assessment Follow-on. Work Package Report 5: Cultural ecosystem services and indicators. UNEP-WCMC, LWEC, UK. http://uknea.unep-wcmc.org/LinkClick.aspx?fileticket=10\%2fZhq\%2bgwtc\%3d\&tabid=82

10

CSIRO (1998). A Guidebook to Environmental Indicators. Commonwealth Scientific and Industrial Research Organisation, Australia. http://www.csiro.au/csiro/envind/

Dallimer, M., Irvine, K.N., Skinner, A.M.J., Davies, Z.G., Rouquette, J.R., Armsworth, P.R., Maltby. L.M., Warren,PH. \& Gaston, K.J. (2012). Biodiversity and the feel-good factor: understanding associations between self-reported human well-being and species richness. BioScience, 62, 47-55.

Environmental Challenge Group (1995). Environmental measures: indicators for the UK environment. London: ECG. 107 pp.

Fagerholm, N. \& Käyhkö, N. (2009). Participatory mapping and geographical patterns of the social landscape values of rural communities in Zanzibar, Tanzania. Fennia, 187, 43-60.

Frank, S., Fürst, C., Koschke, L., Witt, A. \& Makeschin, F. (2013). Assessment of landscape aesthetics - Validation of a landscape metrics-based assessment by visual estimation of the scenic beauty. Ecological Indicators, 32, 222-231.

Fuller, R.A., Irvine, K.N., Devine-Wright, P., Warren, P.H. \& Gaston, K.J. (2007). Psychological benefits of greenspace increase with biodiversity. Biology letters 3, 390-394.

Handley, J., Pauleit, S., Slinn, P., Lindley, S. Baker, M., Barber, A \& Jones, C. (2003). English Nature Research Report 526: Accessible Natural Green Space Standards in Towns and Cities: A Review and Toolkit for their Implementation. Peterborough.

Haines-Young, R. and M. Potschin (2013). Common International Classification of Ecosystem Services (CICES). Report to the European Environment Agency (download: www.cices.eu).

Hernández-Morcillo, M., Plieninger, T. \& C. Bieling (2013). An empirical review of cultural ecosystem service indicators. Ecological Indicators, 29, 434-444.

Higgs, G., Fry R. \& M. Langford (2012). Investigating the implications of using alternative GISbased techniques to measure accessibility to green space. Environment and Planning $B$ : Planning and Design, 39, 326-343. 
Jorgensen, A. \& Anthopoulou, A. (2007). Enjoyment and fear in urban woodlands - Does age make a difference? Urban Forestry \& Urban Greening, 6, 267-278.

Keniger, L.E., Gaston, K..J., Irvine, K.N. \& Fuller, R.A. (2013). What are the Benefits of Interacting with Nature? International Journal of Environmental Research and Public Health, 10, 913-935.

Klain, S.C. and Chan, K.M.A. (2012). Navigating coastal values: participatory mapping of ecosystem services for spatial planning. Ecological Economics, 82, 104-11.

MA (Millennium Ecosystem Assessment) (2005). Ecosystems and Human Well-being: Synthesis. Washington, DC: Island Press.

Martín-López, B., Gómez-Baggethun, E., Lomas \& P.L. \& Montes, C. (2009). Effects of spatial and temporal scales on cultural services valuation. Journal of Environmental Management, 90, 1050-1059.

Morgan, R. (1990). Preferences and Priorities of Recreational Beach Users in Wales, UK. Journal of Coastal Research, 15, 653-667.

Moseley, D., Marzanoa, M., Chetcutia, J. \& Watts, K (2013). Green networks for people: Application of a functional approach to support the planning and management of greenspace. Landscape and Urban Planning 116 1- 12.

Nahuelhual, L., Carmona, A., Lozada, P., Jaramillo, A. \& Aguayo, M. (2013). Mapping recreation and ecotourism as a cultural ecosystem service: An application at the local level in Southern Chile, Applied Geography, 40, 71-82.

Natural England (2011a). Monitor of Engagement with the Natural Environment. Retrieved May 1, 2011, from http://www.naturalengland.org.uk/ourwork/enjoying/research/monitor/

Natural England (2011b). NECR122 - Monitor of Engagement with the Natural Environment: The national survey on people and the natural environment - Technical Report from the 2012-13 survey). See: http://publications.naturalengland.org.uk/publication/1708625 (Retrieved October $16^{\text {th }}, 2013$ )

Office for National Statistics (2013) Quality and Methodology Information: 2011 Census Statistics for England and Wales: March 2011. Downloaded from: http://www.ons.gov.uk/ons/guide-method/census/2011/census-data/2011-census-userguide/quality-and-methods/index.html

Plieninger, T., Dijks, S., Oteros-Rozas, E. \& C. Bieling (2013). Assessing, mapping, and quantifying cultural ecosystem services at community level. Land Use Policy, 33, 118- 129. 
1 Rey Benayas, J.M., Newton, A.C., Diaz, A., Bullock, J.M. (2009). Enhancement of biodiversity 2 and ecosystem services by ecological restoration: a meta-analysis. Science 325 (5944), 3 1121-1124.

4

5 Satz, D., Gould, R.K., Chan, K.M., Guerry, A., Norton, B., Satterfield, T., Halpern, B.S., Levine, 6 J., Woodside, U., Hannahs, N., Basurto ,X. \& Klain, S. (2013). The challenges of incorporating 7 cultural ecosystem services into environmental assessment. Ambio, 42, 675-84.

Schirpke, U., Tasser, E. \& Tappeiner, U. (2013). Predicting scenic beauty of mountain regions. Landscape and Urban Planning, 111, 1-12.

Tengberg A., Fredholm S. , Eliasson I. , Knez I., Saltzman K. \& Wetterberg, O. (2012). Cultural ecosystem services provided by landscapes: Assessment of heritage values and identity. Ecosystem Services, 2, 14-26.

15

van Berkel, D.B. \& Verburg, P. H. (2012). Spatial quantification and valuation of cultural ecosystem services in an agricultural landscape. Ecological Indicators, 37, 163-174.

18 http://dx.doi.org/10.1016/j.ecolind.2012.06.025

19

van Herzele, A. \& Wiedemann, T. (2003). A monitoring tool for the provision of accessible and attractive urban green spaces. Landscape and Urban Planning, 63, 109-126.

Williams, R. (1983). Keywords: a vocabulary of culture and society $\left(2^{\text {nd }} E d.\right)$. Fontana, 24 London. 